03.4

\title{
Численно-экспериментальное исследование деформации пузырька на поверхности в сдвиговом потоке вязкой жидкости
}

\author{
( Ю.А. Питюк, С.П. Саметов, А.И. Муллаянов, О.А. Абрамова
}

Центр микро- и наномасштабной динамики дисперсных систем, Башкирский государственный университет, Уфра, Россия E-mail: pityukyulia@gmail.com

Поступило в Редакцию 26 июля 2019г.

В окончательной редакции 22 августа 2019г.

Принято к публикации 26 августа 2019 г.

\begin{abstract}
Разработан экспериментально-численный подход для изучения деформаций пузырька на поверхности в сдвиговом потоке вязкой жидкости. Численный подход основан на методе граничных элементов для течений Стокса. Для экспериментального изучения деформации пузырька применялись методы оптической микроскопии и высокоскоростной видеосъемки. Исследована динамика изменения отступающего и наступающего контактных углов в зависимости от интенсивности сдвигового потока вязкой жидкости. Получено качественное и количественное согласование результатов численного моделирования и эксперимента для различных капиллярных чисел.
\end{abstract}

Ключевые слова: динамика пузырька, контактный угол, метод граничных элементов, высокоскоростная съемка, оптическая микроскопия.

DOI: 10.21883/PJTF.2019.23.48715.18000

Исследования динамики микропузырьков давно проводятся в России и за рубежом, и уже накоплен значительный объем знаний в этой области. Однако существует множество интересных и важных явлений, которые до конца не изучены. Например, изучение свойств течения вязкой жидкости с пузырьками газа в пористых структурах является важной задачей для развития технологий производства полимерных композитных материалов. Пузырьки в таком случае возникают естественным образом в результате захвата полостей на фронте пропитки [1]. Существуют также технологии использования пузырьков для очистки поверхностей от микро- и нанозагрязнений [2]. Развитие подобных технологий требует детального изучения динамики пузырьков, находящихся в контакте с поверхностью, в частности при воздействии внешних полей.

Экспериментальное исследование особенностей поведения осциллирующего пузырька, находящегося в контакте с поверхностью, в акустическом поле представлено в [3], где описаны особенности возмущения поверхностных мод пузырька, включая формирование струи. Авторы настоящей работы повторили подобный эксперимент, выполнили экспериментально-численное исследование развития колебаний поверхности пузырька в акустическом поле и провели качественное сопоставление результатов численного моделирования и эксперимента [4].

Численное исследование динамики одиночного пузырька в сдвиговом потоке на основе уравнения Навье-Стокса представлено в работах [5,6]. В [5] анализировалось влияние инерциальных, вязких, поверхностных и гравитационных сил на поведение и деформацию пузырька в вертикальном сдвиговом потоке. Авторы работы [6] исследовали поведение пузырька с учетом образования струи и распада на мелкие пузырьки в трехмерном случае.

Несмотря на то что существует множество работ, посвященных исследованию поведения пузырька вблизи твердой стенки и при контакте с ней, особенности динамики пузырька в вязких жидкостях мало изучены. Для восполнения этого пробела в настоящей работе предложен комплексный подход, объединяющий экспериментальные и численные методы.

Численный подход основан на методе граничных элементов для течений Стокса [7] и успешно применялся авторами настоящей работы для расчета динамики сжимаемых пузырьков в неограниченной области в [8], где для замыкания гранично-интегральных уравнений разработан новый подход с применением принципа взаимности Лоренца. Модификация модели для исследования динамики пузырька на поверхности с движущейся и закрепленной контактной линией в случае медленных течений под действием акустического поля представлена нами в [9]. В настоящей работе предложенный подход преобразован для случая сдвигового течения на бесконечности.

Для проведения экспериментальных исследований была разработана и собрана лабораторная установка (рис. 1) на базе длиннофокусного оптического микроскопа и высокоскоростной видеокамеры со скоростью записи изображений в $1000 \mathrm{fps}$ [10]. Основной частью установки является проточная экспериментальная ячейка из прозрачного оргстекла с внутренними размерами $71 \times 7 \times 7 \mathrm{~mm}$. Внутрь ячейки устанавливается лабораторное стекло, поверхность которого была гидрофобизирована раствором диметилдихлорсилана в толуоле (1:10 по объему) [11]. Контактный угол сма- 


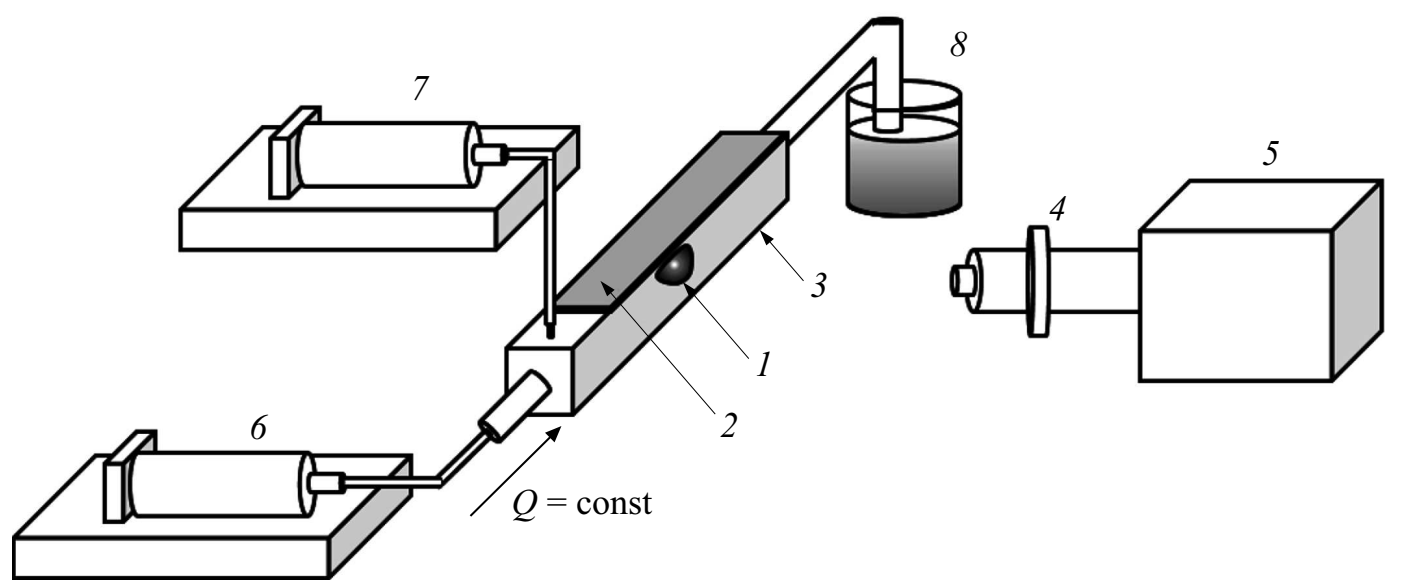

Рис. 1. Схема экспериментальной установки. 1 - пузырь, 2 - гидрофобизированная стеклянная пластина, 3 - экспериментальная ячейка, 4 - длиннофокусный микроскоп, 5 - высокоскоростная камера, 6 - шприцевой насос с глицерином, 7 - шприцевой насос с воздухом, $8-$ сливной сосуд.

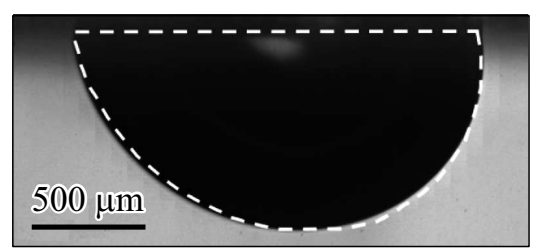

$\mathrm{Ca}=0.21$

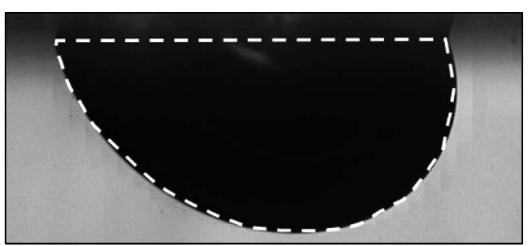

$\mathrm{Ca}=0.32$

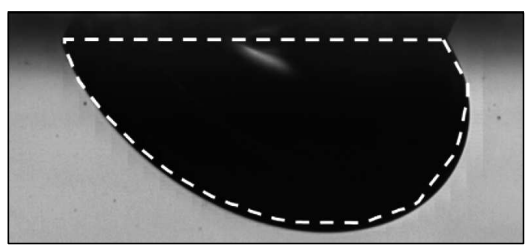

$\mathrm{Ca}=0.5$

Рис. 2. Качественное сравнение форм пузырька, полученных в ходе эксперимента (темный фон) и численного моделирования (светлая штриховая линия), в момент безразмерного времени $t / T=0.2$ для капиллярных чисел Са $=0.21,0.32$ и 0.5 .

чивания на обработанной поверхности с воздушным пузырьком составлял $96^{\circ}$. В качестве рабочей жидкости использовался глицерин вязкостью $1 \mathrm{~Pa} \cdot \mathrm{s}$. Сдвиговый поток жидкости в ячейке создавался шприцевым насосом 200-CE (Cole-Parmer) при объемном расходе в диапазоне $15-45 \mathrm{ml} / \mathrm{min}$. Пузырь воздуха диаметром 1.9-3.8 mm выдувался через иглу с внешним диаметром $0.25 \mathrm{~mm}$. Все эксперименты проводились при температуре $25 \pm 1{ }^{\circ} \mathrm{C}$. Координаты вершин наступающего и отступающего углов, контактные углы смачивания определялись на основе цифровой обработки изображений из видеоряда с помощью программы ImageJ. Методика позволяет оценивать значения контактных углов с погрешностью $\pm 2^{\circ}$, а координаты вершин - c погрешностью $20 \mu \mathrm{m}$.

В результате экспериментальных исследований получены изображения профилей деформируемых пузырьков воздуха, прикрепленных к гидрофобной поверхности, в сдвиговом потоке глицерина. Для численного моделирования была сгенерирована сетка, соответствующая экспериментально полученным начальным формам пузырьков с лапласовским контактным углом порядка 96․ Поскольку модель Стокса в отличие от потенциальных течений [4] требует явного задания твердой поверхности, использовалась геомет- рия в форме сферической шляпы [9]. В эксперименте для каждого капиллярного числа было обработано порядка 30 изображений, в численном моделировании рассматривался 1001 шаг по времени. В математической модели пренебрегается инерцией жидкости и рассматривается сдвиговая скорость на бесконечности в неограниченной области, поэтому характерные времена реального и смоделированного процессов существенно различаются. В связи с этим для сопоставления результатов эксперимента и численного моделирования время отнесено к характерному времени процессов $T$. Эксперимент и численное моделирование проводились в диапазоне капиллярных чисел $\mathrm{Ca} \approx 0.1-0.5$, которые определялись по формуле $\mathrm{Ca}=\mu U / \gamma$, где $U=G a$ - скорость, зависящая от скорости сдвига $G$ и радиуса пузырька $a ; \mu$ - динамическая вязкость жидкости; $\gamma-$ коэффициент поверхностного натяжения.

На рис. 2 представлено качественное согласование форм пузырька, полученных при численном моделировании и из эксперимента, для капиллярных чисел $\mathrm{Ca}=0.21,0.32$ и 0.5 в момент безразмерного времени $t / T=0.2$. Для данного набора капиллярных чисел проведен также анализ динамики контактных углов смачивания, изменения длины контактной линии пу- 

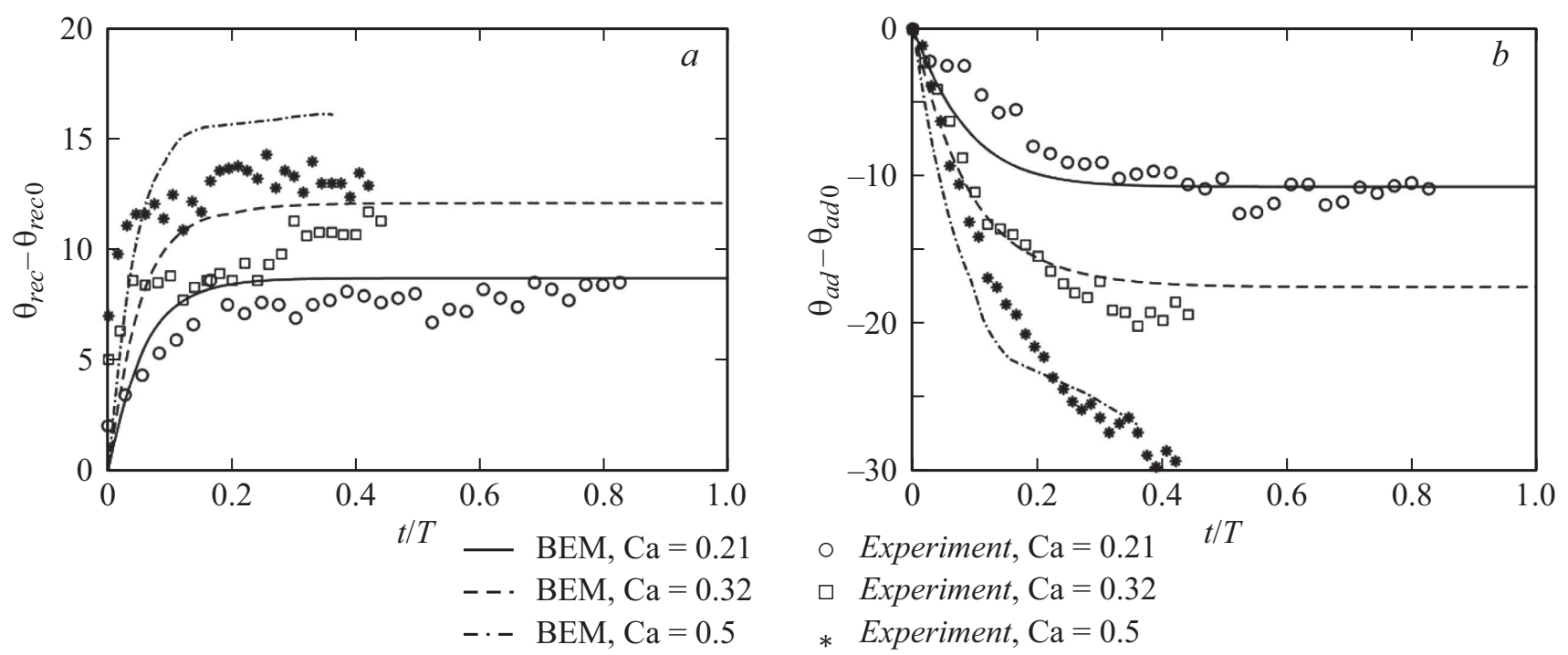

Рис. 3. Изменение отступающего $(a)$ и наступающего $(b)$ контактных углов смачивания пузырька от безразмерного времени $t / T$ для капиллярных чисел $\mathrm{Ca}=0.21,0.32$ и 0.5. Символами показаны экспериментальные точки, линиями - результаты математического моделирования методом граничных элементов (BEM).

зырька и поступательного движения пузырька вдоль поверхности. Изменение отступающего $\left(\theta_{\text {rec }}\right.$ — „receding contact angle“, левый) и наступающего $\left(\theta_{a d}\right.$ — ,advancing contact angle“", правый) контактных углов представлено на рис. 3, $a$ и $b$ соответственно. Из рисунка видно, что для наступающего контактного угла результаты численного моделирования методом граничных элементов хорошо согласуются с экспериментальными данными. Однако изменение отступающего контактного угла, определенное исходя из эксперимента, меньше, чем смоделированное, на $1-3^{\circ}$. Это можно объяснить тем, что скорость потока перед пузырьком больше, чем за ним. Таким образом, точки контактной линии отступающего угла движутся быстрее, чем точки контактной линии наступающего угла. При этом следует отметить, что относительное изменение длины контактной линии для самой большой скорости потока, соответствующей $\mathrm{Ca}=0.5$, не превышает $10 \%$, что позволяет использовать математическую модель с закрепленной контактной линией для анализа контактных углов и форм пузырька.

Таким образом, в работе получено качественное согласование формы пузырьков и изменения динамических контактных углов в результате численного моделирования и эксперимента для различных капиллярных чисел. Полученные результаты можно использовать для решения широкого класса задач, связанных с динамикой микропузырьков на твердых поверхностях, решение которых имеет важное значение для разработки технологий увеличения нефтеотдачи, производства композитных материалов, разработки медицинских, био-, микротехнологий и многих других.

\section{Финансирование работы}

Работа выполнена при финансовой поддержке Российского фонда фундаментальных исследований в рамках научного проекта № 18-38-20102.

\section{Конфликт интересов}

Авторы заявляют, что у них нет конфликта интересов.

\section{Список литературы}

[1] Michaud V. // Transport Porous Media. 2016. V. 115. N 3. P. 581-601. https://doi.org/10.1007/s11242-016-0629-7

[2] Frommhold P.E., Mettin R., Holsteyns F., Lippert A. Surface cleaning by soft acoustic cavitation bubbles // On-line Proc. of Int. Conf. „German Annual Conference on Acoustics“ (DAGA 2012). P. 455-456.

[3] Prabowo F., Ohl C.-D. // Ultrason. Sonochem. 2011. V. 18. N 1. P. 431-435. https://doi.org/10.1016/j.ultsonch.2010.07.013

[4] Абрамова О.А., Ахатов И.Ш., Гумеров Н.А., Питюк Ю.А., Саметов С.П. // Изв. РАН. Механика жидкости и газа. 2018. № 3. C. $3-13$. https://doi.org/10.7868/S0568528118030015

[5] Ervin E.A., Tryggvason G. // J. Fluids Eng. 1997. V. 119. N 2. P. 443-449. https://doi.org/10.1115/1.2819153

[6] Dabiri S., Sirignano W.A., Joseph D.D. // J. Fluid Mech. 2010. V. 651. P. 93-116. https://doi.org/10.1017/S0022112009994058

[7] Pozrikidis C. Boundary integral and singularity methods for linearized viscous flow. Cambridge University Press, 1992. 259 p. https://doi.org/10.1017/CBO9780511624124 
[8] Itkulova Y.A., Abramova O.A., Gumerov N.A. // ASME 2013 Int. Mechanical Engineering Congress and Exposition. American Society of Mechanical Engineers, 2013. P. V07BT08A010-V07BT08A010. http://dx.doi.org/10.1115/IMECE2013-63200

[9] Питюк Ю.А., Гумеров Н.А., Абрамова О.А., Ахатов И.Ш. // Мат. моделирование. 2017. Т. 29. № 9. С. 7789.

[10] Gumerov N.A., Akhatov I.S., Ohl C.D., Sametov S.P., Khazimullin M.V., Gonzalez-Avila S.R. // Appl. Phys. Lett. 2016. V. 108. N 13. P. 134102. https://doi.org/10.1063/1.4944893

[11] Kronberg B., Holmberg K., Lindman B. Surface chemistry of surfactants and polymers. Wiley Online Library, 2014. 479p. http://dx.doi.org/10.1002/9781118695968 